\title{
EFFECTIVENESS OF A HEADS-UP ADAPTIVE LANE DEVIATION WARNING SYSTEM FOR MIDDLE-AGED \& OLDER ADULTS
}

\author{
Nazan Aksan ${ }^{1}$, Lauren Sager ${ }^{1}$, Benjamin Lester ${ }^{1}$, Sarah Hacker ${ }^{1}$, Jeffrey Dawson ${ }^{1}$, \\ Steven W Anderson ${ }^{1}$ \& Matthew Rizzo ${ }^{1,2}$ \\ University of Iowa \& University of Nebraska Medical Center \\ Iowa City, IA, USA \\ nazan-aksan@uiowa.edu
}

\begin{abstract}
Summary: 46 participants ( 24 younger and 22 older) completed at least one out of four simulated drives designed to test the effectiveness of an Adaptive Lane Deviation Warning (LDW) system, and they drove through both a warnings-on and warnings-off version of each drive. Findings showed that LDW was effective in reducing reaction time for lane deviation corrections for both older (by 1.2 seconds) and younger drivers (by 1.6 seconds). The older and younger drivers did not differ in correction RTs when the warnings were turned off. But older drivers showed slower correction RTs than younger drivers in the warning-on drives. The data indicate that these benefits were specific to LDW rather than general improvement in driving performance. Cognitive processing speed emerged as a particularly robust predictor of benefits from the LDW compared to other domains of cognitive function.
\end{abstract}

\section{INTRODUCTION}

Advanced driver assist systems (ADAS) include a variety of warnings for specific driving challenges such as backing, forward-collision, lane departure, blind spot monitoring among others. They began to emerge in early 2000's in luxury models of passenger vehicles. Many current high volume models, increasingly offer more of these systems and NHTSA is considering mandating their inclusion in vehicles (Telematics, 2013, Telematics Update 2014). An important scientific question with public health relevance is how much improvement in driving safety is actually realized with ADAS systems.

The answer to this question must necessarily be population specific. Teenagers and elderly are at increased risk for crashes (NHTSA, 2012). The impact of these systems on safety may vary with age. ADAS may be particularly important to extending safe mobility of the older drivers. On the other hand, age related cognitive decline could conceivably reduce an older driver's ability to respond appropriately to ADAS warnings or even distract the driver from ongoing vehicle operation. To address this issue, we used data from an ongoing study designed to assess the effectiveness of an adaptive, graded warning system for lane departure warning (LDW) among middle-aged and elderly drivers with a range of cognitive impairments.

An informative field study evaluating the effectiveness of a prototype integrated vehicle-based safety (Nodine et al., 2011) showed that driver safety improved from a 12-day baseline period when the warning system was turned off to a 28-day period when the warning system was turned on. For example, LDW was associated with fewer lane departures and increased turn-signal use. While the results of this study were encouraging, there were several limitations. Practice effects 
could not be separated from system benefits in this study as the order of exposure from baseline to driving with warnings was necessarily the same for all subjects for an observational field study. The study did not find negative behavioral adaptation effects over the period observed for any age group. The absence of negative behavioral adaptation effects in particular for older drivers is not consistent with other studies which report greater interference among older subjects when adapting to new technologies (Lavie \& Meyer, 2010). It is also possible lack of negative behavioral adaptation reflects high functioning nature of the elderly recruited into the field study, which may not be representative of the population of older drivers on the road. As noted by Meyer (2009) and Rizzo (2011), individual differences in functional declines associated with aging are large. Research into effectiveness of ADAS would benefit from considering these large differences in cognitive function among elderly (Anstey et al., 2005). While high functioning elderly may adapt to these systems quickly, those with impairments may not derive the intended benefits due to difficulty understanding the warning system messages (Meyer, 2009).

\section{Current Study}

We addressed those limitations in a comprehensive study of warning system effectiveness in middle-aged and older drivers with a range of cognitive impairments for three separate systems: forward-collision, lane change/ merge, and lane departure. The warning systems were graduated and were not designed to replicate the industry standards in important ways. We believe an effective warning system especially for older adults must incorporate the following 3 principles: 1) has both advisory \& imminence features, 2) visuals are heads-up displays, 3) visual displays are distinct from real objects in the scene to minimize confusion, 4) displays do NOT adapt to the scenery $\&$ thus requiring the driver to interpret the warning in context (e.g. warning displays do not change orientation to follow lane markers on curved roadways). But they do change 'message' from advisory to imminent (e.g. change color, add audio, make visual display larger) in simple ways and consistently. In this report, we only evaluated the effectiveness of Lane Departure Warning (LDW; illustrated Figure 1).
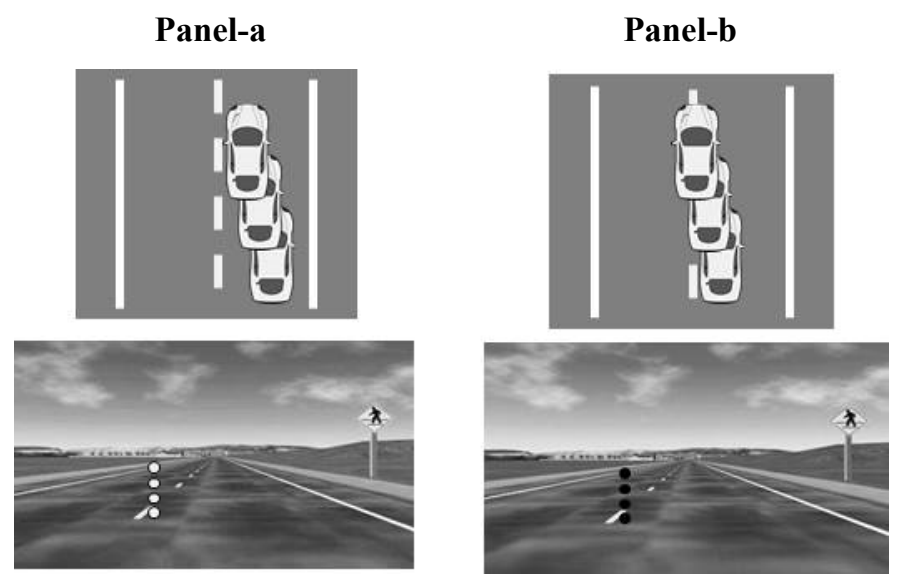

Panel-a depicts the advisory LDW on the left side (yellow circles) as subject begins drifting from the center of the lane. Panel-b depicts the imminent LDW on the left side (red circles) as the vehicle tire overlaps or crosses over the lane markers. The system was turned off when participants activated their turn signal indicating intent to change lanes.

Figure 1. Illustration of LDW visuals (bottom) and the subject's corresponding lane position (top)

A yellow-advisory warning signal (Panel-a of Figure 1) was activated when the driver drifted toward an adjacent lane. A red-imminent warning signal (Panel-b of Figure 1) was triggered 
when the driver's tires overlapped or crossed the lane marker into an adjacent lane. These signals remained activated as long as either of the above conditions was met. Figure 1 shows these visual alerts are distinct from the background scene to improve perception by drivers of all ages. The signals appeared in the lower half of the visual display to encourage drivers to sustain their attention on the roadway. To address LDW effectiveness three questions were asked: 1) Are RTs for lane deviation correction reduced when warnings are activated than when not, and what is the magnitude of safety gains? 2) Do correction RTs in the warnings-on and warnings-off drives differ for older and younger drivers? 3) Are the effects of LDW specific to lane keeping or do they generalize to other safety behaviors such as speed control, not specifically targeted by warning systems we implemented?

Cognitive function measures improve predictions of older driver safety beyond those based on age and basic visual sensory function (reviewed in Anstey et al., 2005). Multiple domains of cognitive function contribute to older driver safety (Rizzo, 2011). Processing speed is a key factor in cognitive aging (Salthouse, 1996) and speed related cognitive declines measured with tools such as the Useful Field of View (UFOV) (e.g. Anstey \& Wood, 2011; Ball et al., 2006) have been related to older driver safety errors. We assessed cognitive functioning in several domains, including speed, visuospatial construction, memory, and executive function (Anderson et al., 2012). We tested if one cognitive domain was more important than others for deriving benefits from LDW than others. For example, speed of processing may be particularly important for RTs to correct lane deviation in the warnings-on drives compared to memory.

\section{METHODS}

\section{Sample}

46 participants (24 younger and 22 older) completed at least one out of four simulated drives. Older drivers ranged from 66 to 87 years $(\mathrm{M}=78.3, \mathrm{SD}=6.6)$ and younger drivers ranged from 25 to 50 years $(M=36.1, S D=8.7)$. Inclusion criteria included: a) valid driver's license, $b)$ minimum 10 years of driving experience, c) driving at least 1 hour or 50 miles per week, d) negative screen for dementia (MoCA > 18, Nasreddine et al., 2005), and e) corrected visual acuity better than $20 / 50$.

\section{Procedure and Design}

Following basic visual function and dementia screening, participants took a simulator drive (fixed base, full cabin with 180 FOV, DriveSafety RS600) to assess motion sickness. Fifteen of 70 tested got motion sick and were not part of the 46 whose data were analyzed here. The remainder participated in detailed assessments of their cognitive function including processing speed, memory, and visuospatial construction during a second visit. In four additional visits scheduled 2 weeks apart, participants took eight simulated drives designed to examine long-term adaptation to warning systems including LDW, forward collision, and lance change/merge. Long-term adaptation to LDW was not addressed in this report. In each of the four visits, participants were exposed to the warnings-on version and warnings-off version of the same drive (i.e. same layout, with same hazards differing only in whether the warnings were turned off or on). The order of presentation was randomized and counterbalanced both with respect to: a) 
whether they experienced the no-warn or the warn version of the drive first in each of four visits b) which of the four distinct drive layouts they first experienced. The drive layouts were similar in terms of tile composition with respect to road culture, total time (15-18 minutes each), and number of hazards encountered.

\section{Measures and Statistical Analyses}

To measure warning system effectiveness, we used average reaction time (RT in seconds) to correct lane deviations from advisory activation to signal off status. This metric was tracked whether the participants actually saw the warnings (warn drive) or did not see the warnings (nowarn drive). High scores on this metric indicate slower corrections. For some of the analyses, this outcome measure was further reduced to a safety gain metric, expressed as the difference in correction RT from no-warn to warn drives. Positive scores on the reduced metric indicate a safety benefit, a score of 0 indicates no benefit from warning system, and negative scores indicate a safety cost due to warnings. $2 \mathrm{X} 2$ mixed design ANOVA with warning status as the within-subject factor (on versus off) and age group as the between subject factor was utilized to evaluate LDW effectiveness using average correction RT across all available data. To evaluate whether the safety benefits of warning systems were specific to the intended effects (e.g. improve lane deviation), average deviation from posted speed limit was used an additional outcome measure. We submitted deviation from speed limit measure to similar statistical analyses concerning no-warn vs. warn differences for older and younger drivers.

Administration and scoring details for cognitive function assessments are described elsewhere (e.g. Lezak et al., 2004). Guided by results of a recent confirmatory factor analysis (Anderson et al., 2012), the raw scores from each test were standardized and averaged to form composite scores quantifying participants relative standing in each domain. Table 1 shows the listing and assignment of each test in our battery to domains of cognitive function. Both bivariate and multivariate associations of cognitive function with correction RTs in the warn drives were examined. Multivariate associations controlled for baseline lane keeping ability by using correction RTs from the no-warn drives, and tested both singly and jointly the contribution of each cognitive domain to prediction of correction RTs in the warn drives.

\section{Table 1. Domains of cognitive function}

Cognitive function domains Tests

Processing Speed Grooved Pegboard, UFOV total score, TMT-Part A

Visuospatial Construction

Complex Figure-Copy, Line Orientation, Block Design

Memory

Controlled Oral Word Association, Complex Figure-Recall,

Auditory Verbal Learning Test-30 minute Recall

Executive Function TMT-Part B, WCST perseveration errors

Abbrv. TMT $=$ Trail Making Test, UFOV $=$ Useful Field of View, WCST $=$ Wisconsin Card Sort

\section{RESULTS}

\section{Warning System Effectiveness}

2x2 mixed design ANOVA showed significant improvement in correction RTs with LDW (main effect of warning condition, $\mathrm{p}<.001$ ). There was a marginally significant main effect of age 
group $(\mathrm{p}<.062)$. Older drivers corrected lane deviations more slowly than younger drivers on average in the warn drives $(\mathrm{p}<.05)$ but not in the no-warn drives $(\mathrm{ns})$. Figure 2, panel-a depicts those effects. The magnitude of the safety gains (difference in correction RT from no-warn to warn drives) was 1.63 seconds $(\mathrm{SD}=1.41)$ for younger and 1.22 seconds $(\mathrm{SD}=2.09)$ for older adults; one-sample t-tests indicated that both of those gains were significantly different than nogain (0-seconds), p's $<.001$. Only 1 out of $24(4.1 \%)$ younger drivers had a negative safety gain score, while 5 out of 22 older drivers $(23 \%)$ had negative safety gain scores. $2 \times 2$ cross-tabulation of age group by whether safety gains were positive or not showed a marginally significant trend indicating that a greater proportion of older drivers than expected failed to show a safety gain $(\mathrm{p}$ $<.10$ Fisher's two-sided exact test). The findings show that LDW speeds lane deviation corrections and the benefits are larger for younger adults.

Figure 2, panel-b depicts parallel analyses conducted for deviation from posted speed limit in nowarn and warn drives. The 2 X2 mixed design ANOVA showed that average deviation in speed did not change from no-warn to warn drives (main effect of warning condition was ns), although younger drivers deviated less than older drivers from the posted speed limit $(p<.005)$ in both nowarn and warn drives (i.e. they drove faster than older drivers). The findings indicate that the effect of warning systems appear specific to lane keeping and not generalized such as improving speed control, an aspect of safety we did not target in this research.

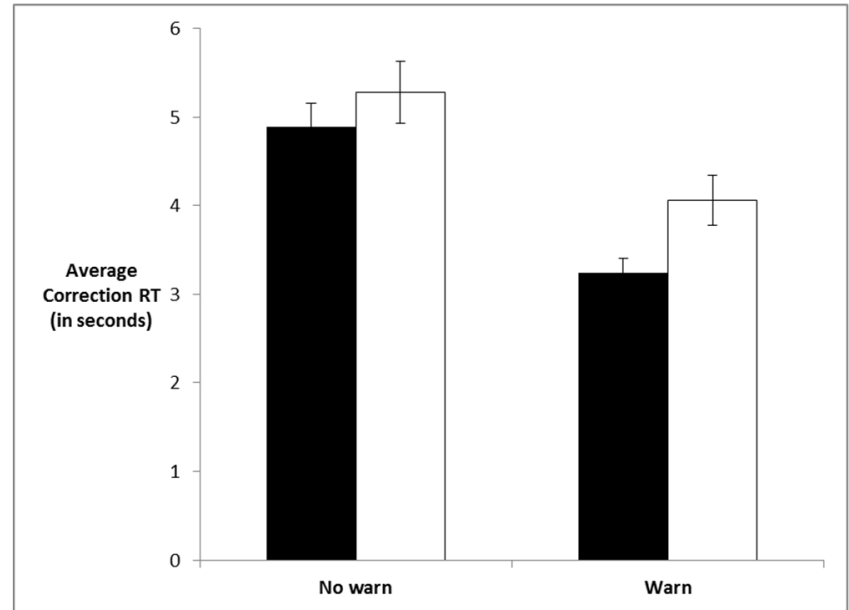

Panel-a

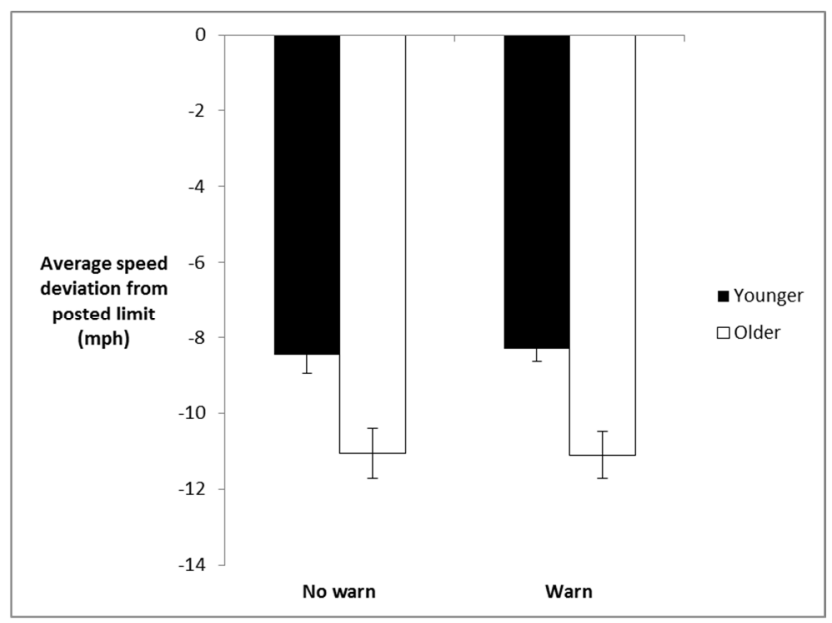

Panel-b

Figure 2. Warning system effectiveness

\section{Associations Of Cognitive Functioning With Warning System Effectiveness}

Table 2 shows the bivariate (Pearson correlations in the first two columns) and multivariate associations (standardized coefficients from multiple regressions in the last two columns) among domains of cognitive function and correction RTs. While all 4 domains of cognitive function were significantly associated with correction RTs in the warn drives, only processing speed was associated with correction RTs in the no-warn drives. Furthermore, only processing speed showed stronger bivariate associations with correction RTs in the warn than no-warn drives, (see p-value column). In addressing whether one domain of cognitive function was a stronger predictor of LDW benefits than others, we controlled for baseline ability in lane keeping in the first step of linear regressions (i.e. correction RTs in the no-warn drives, which were positively 
correlated with correction RTs in warn drives $\mathrm{r}(46)=.432, \mathrm{p}<.001)$. After controlling for baseline lane-keeping, only processing speed added to the prediction of correction RTs in warn drives whether considered singly or jointly with cognitive functioning in other domains.

Table 2. Bivariate correlations and standardized regression coefficients of domains of cognitive function with correction RTs for lane deviations in no-warn and warn drives

\begin{tabular}{|c|c|c|c|c|c|}
\hline \multirow[b]{2}{*}{$\begin{array}{l}\text { Domains of } \\
\text { Cognitive Function: }\end{array}$} & \multicolumn{2}{|c|}{ Correction RT } & \multirow[t]{2}{*}{ p-value ${ }^{a}$} & \multirow{2}{*}{$\begin{array}{l}\text { Std Beta }{ }^{\mathrm{b}} \\
\text { Adjusting for baseline } \\
\text { lane keeping ability only }\end{array}$} & \multirow{2}{*}{$\begin{array}{c}\text { Std Beta }^{\mathrm{c}} \\
\text { Adjusting for both } \\
\text { baseline lane keeping } \\
\& \text { other } \\
\text { cognitive functions }\end{array}$} \\
\hline & No-warn & Warn & & & \\
\hline Speed of processing & $-.344 *$ & $-.602 * *$ & .028 & $-.514 * *$ & $-.870 * *$ \\
\hline Visuospatial construction & $-.274+$ & $-.362 *$ & .28 & $-.264+$ & -.017 \\
\hline Memory & -.185 & $-.362 *$ & .127 & -.292 & .071 \\
\hline Executive function & $-.277+$ & $-.355^{*}$ & .305 & -.254 & .387 \\
\hline
\end{tabular}

Abbrv. Std $=$ Standardized. Listwise $\mathrm{N}=45 ;+\mathrm{p}<.10, * \mathrm{p}<.05, * * \mathrm{p}<.01$ or better. ${ }^{\mathrm{a}} \mathrm{p}$-values indicate whether the correlations of cognitive functioning with correction RTs were stronger in warn than no-warn drives. ${ }^{b}$ Standardized Betas show the prediction to correction RTs in warn drives from each cognitive functioning domain after adjusting for correction RTs in the no-warn drives (numerator $\mathrm{df}=2$ ). ${ }^{\mathrm{c}}$ Standardized Betas show the prediction to correction RTs in warn drives after adjusting for both baseline lane keeping and all other cognitive functioning scores (numerator $\mathrm{df}=5$ ).

\section{DISCUSSION}

Findings showed that LDW was effective in reducing reaction time for lane deviation corrections for both older (a benefit of 1.2 seconds) and younger drivers (a benefit of 1.6 seconds). The warnings benefited younger more than older drivers both in terms of average difference in RTs and proportion showing safety gains. The benefits of LDW were specific to lane keeping in that driving safety indices such as speed control, which were not targeted by the warning systems, did not improve. Because older drivers were slower than younger drivers on average, lack of improvement in speed control from no-warn to warn drives favors an interpretation of specificity in the intended benefits of LDW rather than lack of sensitivity of the drive scenarios to speed control. Findings suggested that cognitive processing speed was a strong predictor of correction RTs when the warnings were on. That prediction remained robust to statistical controls involving both baseline lane keeping ability and cognitive function in other domains. The graduated LDW system implemented in the current study was designed with the limitations of aging drivers in mind. Admittedly, the system we implemented differs from the industry standards in significant ways. However, we did not set out to test a particular commercial system. Collectively, the findings of this study show that even when aging related limitations are considered in warning designs, higher functioning older drivers benefitted more from warnings.

\section{ACKNOWLEDGEMENTS}

We acknowledge the following colleagues for their assistance in data collection and recruitment: Tara Ohrt, Jessica Ferdig, Lacy Flanagan, and Amanda Farmer; Doug Evans (CEO) of Drive Safety for sharing his lane-position tracking code during the initial conceptualization of LDW visuals; the input of Dr. Jim Foley and Dr. Kazu Ebe during the piloting phase of the warning systems. This research was supported by Toyota CSRC. 


\section{REFERENCES}

Anderson, S., Aksan, N, Dawson, J., Uc, Ergun, Johnson, AM. \& Rizzo, M. (2012).

Neuropsychological Assessment of Driving Safety Risk in Older Adults With and Without Neurologic Disease. Journal of Experimental and Clinical Neuropsychology, 34, 895-905.

Anstey KJ, Wood J, Lord S, Walker JG. (2005). Cognitive, sensory and physical factors enabling driver safety in older adults. Clinical Psychology Review, 25, 45-65.

Anstey, KJ \& Woods, J. (2011). Chronological Age and Age-Related Cognitive Deficits Are Associated With an Increase in Multiple Types of Driving Errors in Late Life. Neuropsychology, 25, 613-621.

Ball K, Roenker SL, Wadley VG, Edwards , J. D. , Roth , D. L. et al., (2006). Can high-risk older drivers be identified through performance-based measures in a department of motor vehicles setting? Journal of American Geriatric Society, 54: 77-84.

Lavie, T., \& Meyer, J. (2010) Benefits and costs of adaptive user interfaces. International Journal of Human-Computer Interfaces, 68 508-524.

Lezak, MD, Howieson, D.B., \& Loring, D.W. (2004). Neuropsychological Assessment (4th ed.). New York: Oxford University Press.

Meyer J. (2009) Designing in-vehicle technologies for older drivers. The Bridge: Linking engineering and Society, 39, 21-26.

National Highway Traffic Safety Administration (2012). Traffic Safety Facts 2012: A compilation of motor vehicle crash data from the fatality analysis reporting system and the general estimates system. DPT HS 812032.

Nasreddine ZS, Phillips NA, Bédirian V, Charbonneau S, Whitehead V, Collin I, et al., (2005). The Montreal Cognitive Assessment (MoCA): A Brief Screening Tool For Mild Cognitive Impairment. Journal of the American Geriatrics Society 53, 695-699.

Nodine, E., Lam, A., Stevens, S., Razo, M. \& Naj, W. (2011) Integrated Vehicle-Based Safety Systems (IVBSS) Light Vehicle Field Operational Test Independent Evaluation. NHTSA Report DOT HS 811 516. Cambridge, MA: Volpe National Transportation Systems Center.

Rizzo, M. (2011). Impaired driving from medical conditions: A 70-year-old man trying to decide if he should continue driving. Journal of the American Medical Association, 305, 1018-1026.

Salthouse, T. A. (1996). The processing-speed theory of adult age differences in cognition. Psychological Review, 103, 403-428.

Telematics update (downloaded October 2014). Weekly Brief: NHTSA mandates rear-view cameras, advances ADAS. http://analysis.telematicsupdate.com/other/weekly-brief-nhtsamandates-rear-view-cameras-advances-adas

Telematics (April, 2013). US: NHTSA to add ADAS to NCAP. http://telematicsnews.info/2013/04/08/us-nhtsa-to-add-adas-to-ncap_a486/ 